\title{
BMJ Open Randomised controlled trial assessing the effect of a technology-assisted gait and balance training on mobility in older people after hip fracture: study protocol
}

\author{
Elvira Maranesi, ${ }^{1}$ Giovanni Renato Riccardi, ${ }^{2}$ Fabrizia Lattanzio, ${ }^{1}$ Mirko Di Rosa, ${ }^{3}$ \\ Riccardo Luzi, ${ }^{4}$ Elisa Casoni, ${ }^{2}$ Nadia Rinaldi, ${ }^{5}$ Renato Baldoni, ${ }^{2}$ Valentina Di Donna, ${ }^{5}$ \\ Roberta Bevilacqua (iD ${ }^{1}$
}

To cite: Maranesi E,

Riccardi GR, Lattanzio F, et al. Randomised controlled trial assessing the effect of a technology-assisted gait and balance training on mobility in older people after hip fracture: study protocol. BMJ Open 2020;10:e035508. doi:10.1136/ bmjopen-2019-035508

- Prepublication history and additional material for this paper are available online. To view these files, please visit the journal online (http://dx.doi. org/10.1136/bmjopen-2019035508).

Received 05 November 2019 Revised 30 April 2020 Accepted 04 May 2020

\section{Check for updates}

(c) Author(s) (or their employer(s)) 2020. Re-use permitted under CC BY-NC. No commercial re-use. See rights and permissions. Published by BMJ.

${ }^{1}$ Scientific Direction, IRCCS INRCA, Ancona, Italy ${ }^{2}$ Clinical Unit of Physical Rehabilitation, IRCCS INRCA, Ancona, Italy

${ }^{3}$ Unit of Geriatric

Pharmacoepidemiology, IRCCS INRCA, Ancona, Italy

${ }^{4}$ Medical Direction, IRCCS INRCA, Ancona, Italy

${ }^{5}$ Clinical Unit of Physical Rehabilitation, IRCCS INRCA, Fermo, Italy

Correspondence to Dr Roberta Bevilacqua; r.bevilacqua@inrca.it

\section{ABSTRACT}

Introduction Deficits in balance and walking ability are relevant risk factors for falls during ageing. Moreover, falls are a risk factor for future falls, strongly associated with adverse health outcomes, such as fear of falling or fractures, particularly, hip fracture. For this reason, the development of prevention tools and innovative rehabilitation strategies is one of the main objectives in geriatrics. Effective interventions to promote hip recovery after hip fracture are characterised by intensive and repetitive movements. One treatment approach is to increase the number of steps during the rehabilitation sessions and to improve the balance and the endurance of the patients in the use of technological devices.

Methods and analysis This randomised controlled trial aimed to evaluate an innovative rehabilitation treatment of elderly patients with hip fractures. A total of 195 patients with hip fractures will be recruited and randomly divided into three groups: traditional rehabilitation programme, traditional rehabilitation programme plus TYM0 system and traditional rehabilitation programme plus Walker View. Assessments will be performed at baseline, at the end of treatment, at 6 months, and at 1 and 2 years after the end of the treatment. Only subjects hospitalised 4 weeks prior to the beginning of the study will be taken into consideration. Twenty treatment sessions will be conducted, divided into three training sessions per week, for 7 weeks. The technological intervention group will carry out 30 min sessions of traditional therapy and $20 \mathrm{~min}$ of treatment with a technological device. The control group will perform traditional therapy sessions, each lasting $50 \mathrm{~min}$. The primary outcomes are risk of falling, gait performance and fear of falling.

Ethics and dissemination The study was approved by the Istituto di Ricerca e Cura a Carattere Scientifica, Istituto Nazionale Ricovero e Cura Anziani Ethics Committee, with identification code number 19014. Trial results will be submitted for publication in journals and conferences. Trial registration number NCT04095338.

\section{INTRODUCTION}

Populations are growing older in countries throughout the world. By 2050, nearly 1.2 billion of the expected 1.5 billion people
Strengths and limitations of this study

- This study is a large, controlled, randomised study that analysed innovative clinical practices to treat hip fracture, designed to improve gait and to reduce the risk of falling.

- To our knowledge, this is the first clinical trial to compare traditional rehabilitation and technologydelivered gait performance and balance training in patients with hip fracture.

- The use of instrumented gait analysis, together with clinical outcomes, is the optimal approach to quantify study results.

- It is the first time that the follow-up lasts 2 years after the end of the treatment, representing both a strength and a risk due to an expected higher dropout rate.

aged 65 years or older will reside in today's low-income regions. Just $22 \%$ of the world's older people will live in what we today call high-income countries. ${ }^{1}$ This demographic transformation will profoundly affect the health and socioeconomic development of all nations.

Balance and deficit in walking are some of the main characteristics of ageing and are considered among the risk factors for falls. ${ }^{23}$ Falls are a risk factor for future falls and are associated with other adverse health outcomes, such as fear of falling or fractures. $^{4-8}$ In particular, hip fractures can have a devastating impact on the ability of older patients to remain independent. Individuals following hip fractures experience greater postural sway, possibly due to reduced muscular strength and proprioception. ${ }^{9}$ Reports show that one in three patients dies within the first year after injury, while survivors have poor quality of life. ${ }^{10}$ 


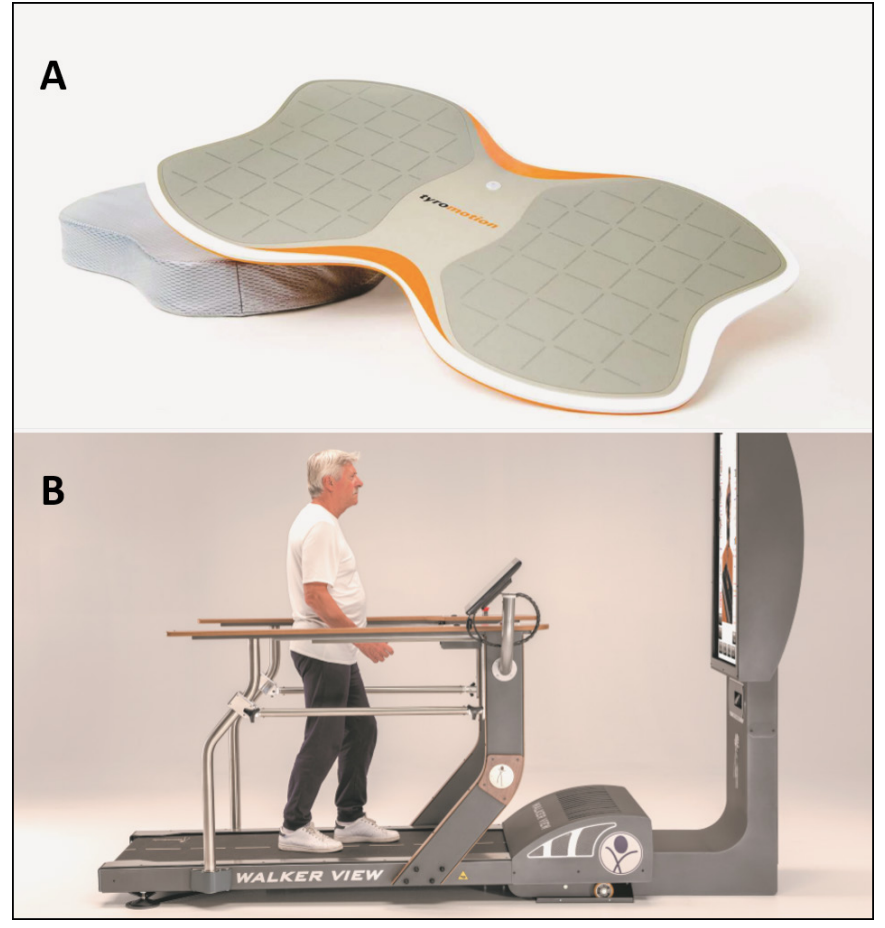

Figure 1 Technological devices used during the rehabilitation treatment: TYMO system $(A)$ and Walker View (B). The image in the article is a commercial one of the Walker View system and not one of our patients.

For these reasons, the development of prevention tools and innovative strategies in the rehabilitation field should be one of the main objectives in the treatment of the diseases afflicting the elderly, such as hip fractures. ${ }^{11}$ Evidence suggests that rehabilitation plays a crucial role in guaranteeing recovery and enhancing quality of life following hip fracture. This pathological condition is routinely treated by rehabilitative approaches aimed at improving the static/dynamic balance, the recovery of walking and the prevention of falls. ${ }^{12-14}$ From the literature, it is evident that there is no standard rehabilitation practice common to all. No standard set of key outcomes or measures was used across the previously designed investigations. The ingredients of programmes and practices evaluated were dissimilar and varied in their intensity, duration and timing of the initiation. ${ }^{15}$

One of the few practices that seems to be effective in hip fracture rehabilitation is a specific task repetitive training since, by increasing therapy dosage, intensity and number of repetitions, the plasticity and the functional recovery are promoted. ${ }^{16} \mathrm{~A}$ treatment approach to increase the number of steps during rehabilitation sessions and to improve balance and endurance of the patients is the use of robotic systems. In this study, the technological devices used for the rehabilitation treatment are the TYMO system (Tyromotion, Austria), a wireless static and dynamic platform for evaluating and rehabilitating posture (figure 1A), or the Walker View (TecnoBody, Italy) a treadmill equipped with a sensorised belt with eight load cells and a 3-D camera (figure 1B).
The two intervention groups will be treated with only one of the two technological devices, combined with traditional therapy.

\section{Study aims and objectives}

This study aimed to evaluate an innovative rehabilitation treatment based on technological devices for older patients with hip fractures, designed to improve gait performance and to reduce risk of falling. The primary aim was to evaluate the effect of the rehabilitation treatment on balance and gait performance of older people with hip fracture, as a result of the use of the TYMO system or the Walker View, at the end of the treatment and at 6 months and 1 and 2 years of follow-up, by using the Performance-Oriented Mobility Assessment (POMA) scale.

The secondary aims were

1. To evaluate the effect of the rehabilitation treatment on gait speed of older people with hip fracture, as a result of the use of the TYMO system or the Walker View, at the end of the treatment and at 6 months and 1 and 2 years of follow-up, by using instrumental gait analysis.

2. To evaluate the effect of the rehabilitation treatment on the fear of falling of older people with hip fracture, as a result of the use of the TYMO system or the Walker View, at the end of the treatment and at 6 months and at 1 and 2 years of follow-up, by using Short Falls Efficacy Scale-International (FES-I) Short Form Scale.

\section{METHODS AND ANALYSIS \\ Trial design}

This study is a randomised, single blind (outcome assessors) controlled trial. A total of 195 patients with hip fractures will be recruited and randomly divided into three groups: (1) traditional rehabilitation programme, (2) traditional rehabilitation programme plus TYMO system and (3) traditional rehabilitation programme plus Walker View. Assessments will be performed at baseline, at the end of treatment, and at 6 months and 1 and 2 years after the end of the treatment. In addition, the study design includes the use of a standardised questionnaire and instrumental gait analysis, in order to collect data on the improvements with a mix-method approach. This methodology is routinely applied in our institute.

\section{Study setting}

The study will be conducted at the Clinical Unit of Physical Rehabilitation, Istituto di Ricerca e Cura a Carattere Scientifica, Istituto Nazionale Ricovero e Cura Anziani (IRCCS INRCA), in the Ancona and Fermo branches, Italy. Assessments and treatments will be conducted in the robotics laboratories. Patients will be selected from the clinic at the two hospitals' physical rehabilitation clinic after receiving the appropriate treatment to be stabilised and discharged.

\section{Patient and public involvement}

No patients were involved. 


\section{Trial status}

At the time of the submission of this study protocol, data collection was ongoing. The first patient was recruitment on 26 November 2019. The study is expected to end in August 2021.

\section{Participants}

The inclusion criteria ${ }^{1217}$ are

- Age 65 years and over.

- Capacity to provide consent.

- Traumatic event within 60 days.

- Romberg test: negative.

- Functional Ambulation Category (FAC) score of $\geq 2$.

- Rankin Scale score of $\leq 3$.

The exclusion criteria are

- Failure to meet the inclusion criteria.

- Concomitant participation in other studies.

- History of syncopal episodes.

- Presence of non-pharmacologically compensated behavioural syndromes.

- Presence of pain that prevents walking or standing.

- Presence of neurological pathologies that compromise balance (multiple sclerosis, Parkinson's disease, stroke, myelosion, ataxias or poliomyelitis).

- Spinal stenosis.

- Radiculopathy.

- Neuropathies in the lower limbs.

- Disabling disabilities that impair walking (eg, congenital malformations of the foot).

- Heterometry of $>2 \mathrm{~cm}$.

- Lack of written informed consent.

- Clinical Dementia Rating (CDR) score of $\geq 3$.

- Severe systemic diseases with life expectancy of $<1$ year.

\section{Sample size}

The POMA, ${ }^{18}$ a test widely used to assess walking ability and associated with equilibrium, was used to calculate the sample size. ${ }^{19}$ Assuming a small effect size of $10 \%,{ }^{20}$ it is estimated that the overall sample size needed to capture this effect size is of 153 subjects, assuming a statistical power of $80 \%$, a significance level of 0.05 , three groups and five repeated assessments (a baseline and four follow-ups) in an analysis of variance (ANOVA) model within-between interactions. Even assuming a $25 \%$ drop-out rate, the total number required would be 195 subjects (65 for each arm).

It is hypothesised that this sample dimension is more than sufficient to grasp a variation also for secondary outcomes for which a treatment effect size is assumed of a similar or higher magnitude than that identified for the primary outcome. ${ }^{1721}$

\section{Recruitment}

Patients are selected by the outpatient department at the Clinical Unit of Physical Rehabilitation, IRCCS INRCA, in the Ancona and Fermo branches. Patients are contacted to schedule a visit with the physician. Once the compliance with the inclusion and exclusion criteria of the study has been verified and informed consent has been obtained in triplicate, the doctor, together with a physiotherapist and a biomedical engineer, proceeds with the baseline evaluation and with acquisition of gait assessment parameters through gait analysis at the Movement Analysis Laboratory of the Clinical Unit of Physical Rehabilitation of the Ancona branch. Functional and cognitive evaluation scale scores are obtained by a physiotherapist and a psychologist, respectively. A copy of the informed consent is reported in online supplementary file 1 .

A randomisation technique based on a single sequence of random assignments is used. A list of random numbers generated by the computer is used and subjects are assigned a number based on their order of inclusion in the study. According to this technique, the 195 subjects will be randomly assigned to one of the three study groups.

At the end of the treatment and after 6 months and 1 and 2 years, patients will be contacted again to schedule subsequent follow-up visits and upgrades.

Recruitment will run from August 2019 to August 2021.

\section{Intervention}

For the study, outpatients are involved 4 weeks after hospitalisation in the Clinical Unit of Physical Rehabilitation, IRCCS INRCA, in the Ancona and Fermo branches. Participants have already received the standard treatment. Twenty treatment sessions will be conducted, divided into three training sessions per week, for 7 weeks. The technological intervention groups, using the TYMO system or the Walker View, carry out $30 \mathrm{~min}$ sessions of traditional therapy, plus 20 min of treatment with a technological device. The control group performs $50 \mathrm{~min}$ traditional therapy sessions. Cardiac activity monitoring is conducted during robotic treatments in order to detect the heart rate during physical activity. ${ }^{22}$

Individual participants must complete at least $80 \%$ of the overall planned sessions. The recovery of two sessions will be possible.

All patients included in the study perform traditional rehabilitation treatments consisting of

- Passive mobilisation for the recovery of the complete articular range.

- Scar removal massage therapy and possible draining massage of the lower limb.

- Muscle strengthening with isometric and isotonic exercises.

- Proprioceptive exercises in standing position for load balancing and balance control.

- Step training with progressive reduction of walking aids.

- Recovery of autonomy in stair ascent and descent.

- Recovery of autonomy in daily life activities.

The technology-delivered gait and balance training consists of using either of two different devices: the TYMO system or the Walker View.

The TYMO system is a wireless platform for balance and the postural control training. The TYMO system is connected to a screen and provides virtual reality games, 


\begin{tabular}{lll}
\hline Table 1 Outcomes and clinical assessments \\
\hline & $\begin{array}{l}\text { Clinical } \\
\text { assessment }\end{array}$ & $\begin{array}{l}\text { Expected } \\
\text { improvement at the } \\
\text { end of treatment (\%) }\end{array}$ \\
\hline $\begin{array}{l}\text { Outcomes } \\
\text { of the overall mobility } \\
\text { (balance+walking ability) }\end{array}$ & 10 \\
$\begin{array}{l}\text { Secondary: improvement of } \\
\text { gait speed }\end{array}$ & $\begin{array}{l}\text { Instrumental } \\
\text { gait analysis }\end{array}$ & 12 \\
$\begin{array}{l}\text { Secondary: decrease of fear } \\
\text { of falling }\end{array}$ & FES-I Short & 15 \\
\hline
\end{tabular}

FES-I, Short Falls Efficacy Scale-International; POMA, PerformanceOriented Mobility Assessment.

adaptable to the functional capacity of the patient. Through the games proposed, the physiotherapist will decide to work in a dimension (anteroposterior or mediolateral) or in two dimensions (combining the anteroposterior and mediolateral movements).

The Walker View is a treadmill equipped with a sensorised belt with eight load cells and a three-dimensional (3-D) camera to detect length, speed and symmetry of the pace and load, range of the trunk, hips and knees. Patients are asked to walk at a comfortable speed while the physiotherapist is able to work on different parameters, such as step length, load distribution and step height. The setting takes into account the clinical conditions of each patient, allowing the customisation of the intervention. The Walker View offers visual and auditory feedback to the patient so they can correct gait in real time. Details of the exercises carried out by the three groups are shown in the online supplementary file 2.

\section{Outcomes}

All outcome measures follow a standardised operating procedure. Table 1 shows the primary outcome and secondary outcomes with the expected result at the end of the treatment. The expected improvement is derived from the analysis of similar studies, ${ }^{20}$ collected for the evaluation of the sample size for each outcome.

Further evaluations will be carried out as follows:

- Length and asymmetry of the step, through instrumental gait analysis.

- Walking and functional status, through the FAC and Barthel Index (BI) Scale.

- Acceptance of the technology, through the Psychosocial Impact of Assistive Device Scale questionnaire.

- Quality of life, through the Short Form (SF)-12 questionnaire.

Mini-Mental State Examination (MMSE): The MMSE was designed as a clinical method for grading cognitive impairment. The score ranges from 0 to 30: scores of $\geq 24$ indicate normality; scores between 18 and 23 indicate mild cognitive impairment; scores between 11 and 17 indicate average cognitive deficits; and scores of $\leq 10$ indicate severe cognitive impairments. The reported score is corrected for age and education. ${ }^{23}$
Rankin Scale: The Rankin Scale is a simple scale for the evaluation of the outcomes following a stroke. Reliability is well defined. The individual categories are essentially based on patient mobility. There are six grades of classification from 0 to 5 , where 0 means independence. ${ }^{24}$

Barthel Index: BI is an ordinal scale used to measure a subject's performance in everyday life activities. The index analyses 10 variables that describe the activities of daily life and mobility. Each item is assigned a score between 0 and 10, depending on the degree of the patient's functionality: full, reduced or no functionality. A high overall score is associated with a greater probability of being able to live at home independently after discharge from the hospital. ${ }^{25}$

Functional Ambulation Category: The scale is used to classify the severity level of gait disturbances in neurological disorders. It provides a hierarchical classification from level 0 (impossible walking) to level 5 (no limitation). ${ }^{26}$ SF-12 Health Survey: The SF-12 questionnaire was originally developed in the USA to provide a short alternative form to the SF-36 questionnaire. The SF-12 is composed of 12 items that produce two measurements related to two different aspects of health: physical health and mental health. The subject is asked to answer on how he feels and how he is able to carry out the usual activities, evaluating the current day and the four previous weeks. ${ }^{27}$

Tinetti's Scale or POMA: The Tinetti scale is a tool used to evaluate balance and gait performance. The test is used clinically to determine the mobility status of a subject or to assess changes in balance and gait time. The total POMA consists of two subscales: the balance evaluation scale ('balance scale' or POMA-B) and the gait evaluation scale ('gait scale' or POMA-G). The maximum score is 28 points: in detail, the maximum score of the POMA-B is 16 , while for the POMA-G, the maximum score is $12 .^{18}$

Short Falls Efficacy Scale-International: The scale measures the 'fear of falling'. The scale can be self-administered or administered during the interview. The cut-offs for the fear of falling are divided as follows: a score of 7-8 indicates low concern; a score between 9 and 12 indicates moderate concern; and a score between 14 and 28 indicates high concern. ${ }^{28}$

Psychosocial Impact of Assistive Device Scale: It is a selfcompletion questionnaire to be completed by the user and it assesses the impact that the device has on the person. Through 26 questions, it tries to detect how the device has brought about a perception of change with respect to one's availability for new experiences (6 questions), skills (ability to cope with daily activities and challenges, 12 questions) and self-esteem (security and self-confidence, 8 questions). Every question is answered on a visual scale from -3 (the device has strongly limited my independence) to +3 (the device has greatly improved my independence).$^{29}$

Assistive Device Predisposition Assessment: The purpose of the tool is to assess user expectations about technological devices. $^{30}$ 


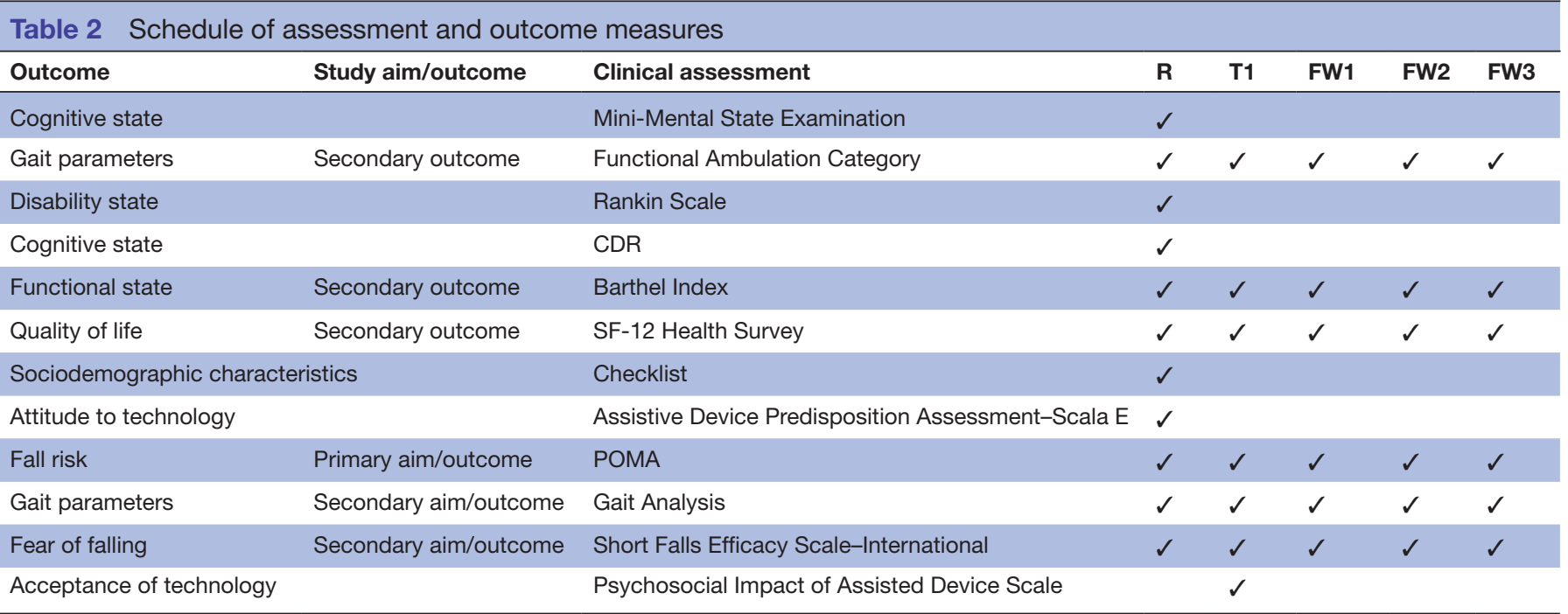

CDR, Clinical Dementia Rating; FW1, first follow-up at 6 months since the end of treatment; FW2, second follow-up at 1 year since the end of treatment; FW3, third follow-up at 2 years since the end of treatment; POMA, Performance-Oriented Mobility Assessment; R, Recruitment; SF, Short Form; T1, end of treatment.

CDR Scale: This questionnaire assesses the patient's dementia status. The CDR is a 5-point scale used to characterise six domains: memory, orientation, judgement and problem solving, business, home and hobby and personal care. ${ }^{31}$

Gait analysis and instrumental postural analysis: Gait analysis is the systematic study of human locomotion, augmented by instrumentation for measuring body movements, body mechanics and the activity of the muscles. ${ }^{32}$ Gait analysis is performed on the selected patients at the Gait Analysis Laboratory in the Department of Physical Rehabilitation at the branch of IRCSS INRCA Ancona. Instrumented gait analysis is performed using BTS GAITLAB (BTS Bioengineering, Italy) system with six infrared cameras $(100 \mathrm{~Hz})$ and two force plates $(50 \mathrm{~Hz})$. The system is used to acquire both kinematic and kinetic data. 3-D kinematic data are recorded with the help of 22 reflective infrared markers using the Helen Hayes protocol. ${ }^{33}$ The floormounted force plates are used to acquire the kinetic data. The subjects walked at a self-selected speed along a straight stretch $7 \mathrm{~m}$ long. The parameters obtained from gait analysis are used to answer the first two objectives of this study protocol, namely, to verify how technological treatment improves gait performance in patients with hip fracture.

A summary of all data collected and when these are collected is provided in table 2 .

\section{Data management}

Personal data collected during the trial will be handled and stored in accordance with the General Data Protection Regulation 2018. The use of the study data will be controlled by the principal investigator. All data and documentation related to the trial will be stored in accordance with applicable regulatory requirements, and access to data will be restricted to authorised trial personnel.

\section{Data analysis}

Continuous variables will be reported as either mean and SD or median and IQR on the basis of their distribution (assessed using Shapiro-Wilk test). Comparison of variables between groups will be performed by unpaired Student t-test or Mann-Whitney U test according to their distribution. Categorical variables will be expressed as absolute numbers and percentage, and statistical significance will be assessed by $\chi^{2}$ test or Fisher exact test.

In a second step, the analysis of the follow-up data will be carried out in order to evaluate the effectiveness of the intervention. This analysis phase will involve the use of multivariate statistical techniques, in particular, repeated measures ANOVA, in order to compare the changes over time in the outcome measures between the intervention group and the control group. The statistical significant will be set at $\mathrm{p}<0.05$.

\section{ETHICS AND DISSEMINATION \\ Ethics}

The study was approved by IRCCS INRCA Ethics Committee during the session of 17 July 2019, with identification code number 19016. Any protocol modifications will be reported to the same ethics committee. The principals of the Declaration of Helsinki and Good Clinical Practice guidelines will be adhered to.

\section{Administrative structures}

The trial will be run by principal investigator and coinvestigators (a physiatrist, a biomedical engineer, a psychologist and a statistician) and two therapists. There is no external funding for the realisation of the study.

\section{Dissemination}

The dissemination programme will involve peer-reviewed journal and national and international conferences. The results will be disseminated to all participants. 
Contributors EM and RBo led the design and writing of the pilot randomised controlled trial protocol. VDD and GRR helped with the development of the participant identification plan and provided advice on other key study issues. EC, NR and RBa helped with the design of the intervention. MDR will lead the collection, management and statistical analysis of the data. FL and RL contributed feedback on the trial design. All the authors contributed important intellectual content to the written protocol and approved the final version for publication.

Funding The authors have not declared a specific grant for this research from any funding agency in the public, commercial or not-for-profit sectors.

Competing interests None declared.

Patient and public involvement Patients and/or the public were not involved in the design, conduct, reporting or dissemination plans of this research.

Patient consent for publication Not required.

Provenance and peer review Not commissioned; externally peer reviewed.

Open access This is an open access article distributed in accordance with the Creative Commons Attribution Non Commercial (CC BY-NC 4.0) license, which permits others to distribute, remix, adapt, build upon this work non-commercially, and license their derivative works on different terms, provided the original work is properly cited, appropriate credit is given, any changes made indicated, and the use is non-commercial. See: http://creativecommons.org/licenses/by-nc/4.0/.

\section{ORCID iD}

Roberta Bevilacqua http://orcid.org/0000-0002-3851-3552

\section{REFERENCES}

1 Kinsella K, Phillips DR. Global aging: the challenge of success. Population bulletin. 60. Washington: Population Reference Bureau, 2005.

2 Johnell O, Kanis JA. An estimate of the worldwide prevalence, mortality and disability associated with hip fracture. Osteoporos Int 2004;15:897-902.

3 Reeve J, Loveridge N. The fragile elderly hip: mechanisms associated with age-related loss of strength and toughness. Bone 2014;61:138-48.

4 Maranesi E, Merlo A, Fioretti S, et al. A statistical approach to discriminate between non-fallers, rare fallers and frequent fallers in older adults based on posturographic data. Clin Biomech 2016;32:8-13.

5 Maranesi E, Fioretti S, Ghetti GG, et al. The surface electromyographic evaluation of the functional reach in elderly subjects. J Electromyogr Kinesiol 2016;26:102-10.

6 Boyd R, Stevens JA. Falls and fear of falling: burden, beliefs and behaviours. Age Ageing 2009;38:423-8.

7 Scheffer AC, Schuurmans MJ, van Dijk N, et al. Fear of falling: measurement strategy, prevalence, risk factors and consequences among older persons. Age Ageing 2008;37:19-24.

8 Vellas BJ, Wayne SJ, Romero LJ, et al. Fear of falling and restriction of mobility in elderly fallers. Age Ageing 1997;26:189-93.

9 McKenzie Al, Briggs RA, Barrows KM, et al. A pilot study examining the impact of exercise training on skeletal muscle genes related to the TLR signaling pathway in older adults following hip fracture recovery. J Appl Physiol 2017;122:68-75.

10 Berry SD, Kiel DP, Colón-Emeric C. Hip fractures in older adults in 2019. JAMA 2019;321:2231-2.

11 Hopewell S, Adedire O, Copsey BJ, et al. Multifactorial and multiple component interventions for preventing falls in older people living in the community. Cochrane Database Syst Rev 2018;7:CD012221.

12 Monticone M, Ambrosini E, Brunati R, et al. How balance taskspecific training contributes to improving physical function in older subjects undergoing rehabilitation following hip fracture: a randomized controlled trial. Clin Rehabil 2018;32:340-51.

13 Monticone M, Ambrosini E, Rocca B, et al. Task-oriented exercises and early full weight-bearing contribute to improving disability after total hip replacement: a randomized controlled trial. Clin Rehabil 2014;28:658-68.

14 Wu J-Q, Mao L-B, Wu J. Efficacy of balance training for hip fracture patients: a meta-analysis of randomized controlled trials. J Orthop Surg Res 2019;14:83.

15 Chudyk AM, Jutai JW, Petrella RJ, et al. Systematic review of hip fracture rehabilitation practices in the elderly. Arch Phys Med Rehabil 2009;90:246-62.

16 Taveggia G, Borboni A, Mulé C, et al. Conflicting results of robotassisted versus usual gait training during postacute rehabilitation of stroke patients: a randomized clinical trial. Int J Rehabil Res 2016;39:29-35

17 van Ooijen MW, Roerdink M, Trekop M, et al. Functional gait rehabilitation in elderly people following a fall-related hip fracture using a treadmill with visual context: design of a randomized controlled trial. BMC Geriatr 2013;13:34.

18 Tinetti ME. Performance-oriented assessment of mobility problems in elderly patients. J Am Geriatr Soc 1986;34:119-26.

19 Faber MJ, Bosscher RJ, van Wieringen PCW. Clinimetric properties of the performance-oriented mobility assessment. Phys Ther 2006;86:944-54.

20 Meurisse GM, Bastien GJ, Schepens B. The step-to-step transition mode: a potential indicator of first-fall risk in elderly adults? PLoS One 2019;14:e0220791.

21 Werner C, Chalvatzaki G, Papageorgiou XS, et al. Assessing the concurrent validity of a gait analysis system integrated into a smart Walker in older adults with gait impairments. Clin Rehabil 2019;33:1682-7

22 Agostinelli A, Morettini M, Sbrollini A, et al. CaRiSMA 1.0: cardiac risk self-monitoring assessment. Open Sports Sci J 2017;10:179-90.

23 Folstein MF, Folstein SE, McHugh PR, et al. "Mini-mental state". A practical method for grading the cognitive state of patients for the clinician. J Psychiatr Res 1975;12:189-98.

24 van Swieten JC, Koudstaal PJ, Visser MC, et al. Interobserver agreement for the assessment of handicap in stroke patients. Stroke 1988;19:604-7.

25 Mahoney FI, Barthel DW. Functional evaluation: the BARTHEL index. Md State Med J 1965;14:61-5.

26 Holden MK, Gill KM, Magliozzi MR. Gait assessment for neurologically impaired patients. standards for outcome assessment. Phys Ther 1986;66:1530-9.

27 Ware JE, Kosinski M, Keller SD. SF-12: how to score the SF-12 physical and mental health summary scales. 3rd edn. Lincoln, RI: QualityMetric Incorporated, 1998.

28 Ruggiero C, Mariani T, Gugliotta R, et al. Validation of the Italian version of the falls efficacy scale International (FES-I) and the short FES-I in community-dwelling older persons. Arch Gerontol Geriatr 2009;49(Suppl 1):211-9.

29 Jutai J, Day H. Psychosocial impact of assistive devices scale (PIADS). Technol Disabil 2002;14:107-11.

30 Scherer MJ, Cushman LA. Measuring subjective quality of life following spinal cord injury: a validation study of the assistive technology device predisposition assessment. Disabil Rehabil 2001;23:387-93.

31 Morris JC. Clinical dementia rating: a reliable and valid diagnostic and staging measure for dementia of the Alzheimer type. Int Psychogeriatr 1997;9(Suppl 1):173-6.

32 Levine DF, Richards J, Whittle M. Whittle's gait analysis. Elsevier health sciences, 2012. ISBN: 978-0702042652.

33 Davis RB, Õunpuu S, Tyburski D, et al. A gait analysis data collection and reduction technique. Hum Mov Sci 1991;10:575-87. 